\title{
Visualization of Asian Soybean Rust (Phakopsora pachyrhizi) Fungal Infections and Cell Ultrastructure to Characterize Fungicide Action In Situ
}

\author{
Heather Pence, Andrew Bowling and Lori Breitweiser
}

Corteva Agriscience, Indianapolis, Indiana, United States

Asian soybean rust (ASR) causes a significant reduction of yield in soybean, causing billions of dollars of damage annually on one of the world's most important crops [1]. Application of fungicides is critical in many geographies today to consistently achieve optimal crop yields. Crop protection fungicides are routinely evaluated with field trials in multiple geographies under a broad range of environmental conditions [2]. While this field trial data is critical to understanding the efficacy and spectrum of a fungicidal molecule, microscopic studies allow for a much deeper understanding of the action of the molecule at the cellular level.

ASR spores first land on a soybean leaf, germinate, and form structures called appressoria, which facilitate the penetration of the outermost layer of the leaf epidermal cells with an infection peg. Once the infection has progressed to the inside of the leaf, confocal microscopy can be used to image deep into the leaf tissue to allow visualization of the developing fungal infection and evaluation of the curative action of applied fungicides. To prepare soybean leaves for confocal imaging, dissected samples were fixed in 3:1 ethanol:acetic acid, treated with $2 \% \mathrm{NaOH}$ at $90 \mathrm{deg} \mathrm{C}$ for 1 hour, and stained with wheat germ agglutininAlexaFluor 488 (WGA-AF488) lectin conjugate. Leaf pieces were cleared with glycerol and imaged on a Leica SP5 LSCM. At 3 days post inoculation (DPI), the ASR infections have grown nearly to the bottom of the leaf (Figure 1A). After 5 DPI, the ASR infection has begun to spread laterally within the leaf (Figure 1B). However, if infected leaves are sprayed with fungicide at 3 DPI and imaged two days later at 5 DPI, the growth of the fungus has been completely halted inside the leaf tissue (Figure 1C).

The fungicide used in this study has stopped the growth and expansion of fungal cells inside the soybean leaf (Figure 1). However, from this confocal data, it is unclear if the fungal cells are living but not able to grow or if they are dead. One way to assess the viability of fungal cells inside an infected leaf is to visualize the cellular ultrastructure using TEM. To prepare soybean leaves for TEM, dissected leaves were fixed with glutaraldehyde and osmium, dehydrated, embedded in LR White resin, and ultrathin sections were cut and mounted on uncoated grids. A fungal appressorium and infection peg penetrating the surface of a soybean epidermal cell (Figure 2A) and fungal hyphae cells ramifying through the leaf can be seen (Figure 2B). At higher magnification, fungal cells inside an untreated leaf have well-defined and easily recognizable organellar structures, such as plasma membranes, ER, nuclei, mitochondria (Figure 2C). However, fungal cells within fungicide-treated leaves show no evidence of organellar structures (Figure 2D). Thus, these results strongly suggest that this particular fungicide has killed the fungal cells in situ within the soybean leaf.

In summary, the WGA staining - leaf clearing - confocal imaging method presented in this study can be used to show the impact of fungicides on the growth of ASR fungal infections. Furthermore, classic thinsection TEM methods can be used to directly determine the viability of fungal cells in plant leaves to assess fungicide action in situ. 

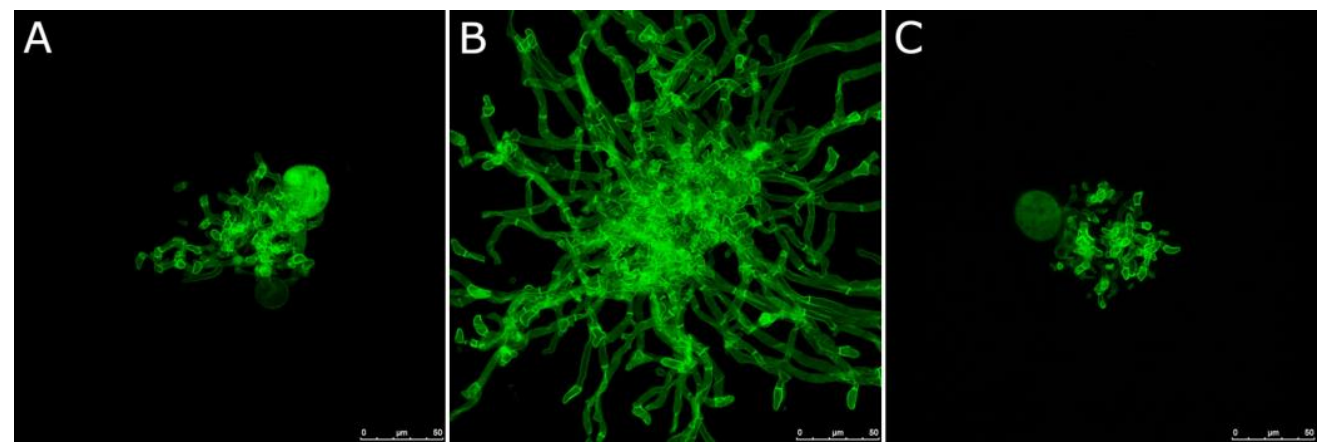

Figure 1. Maximum projection confocal images of ASR-infected soybean leaves stained with WGAAF488. A.) A fungal infection inside an untreated soybean leaf at 3 DPI, showing a relatively tight mass of fungal hyphae cells. B.) A fungal infection inside an untreated soybean leaf at 5 DPI. The fungal infection has grown significantly after two days when left untreated. C.) A fungal infection inside a soybean leaf that was sprayed with fungicide at 3 DPI and collected for imaging two days later at 5 DPI. The size/extent of the fungal infection appears very similar to untreated infections at $3 \mathrm{DPI}$, as if no further growth has occurred following fungicide application.

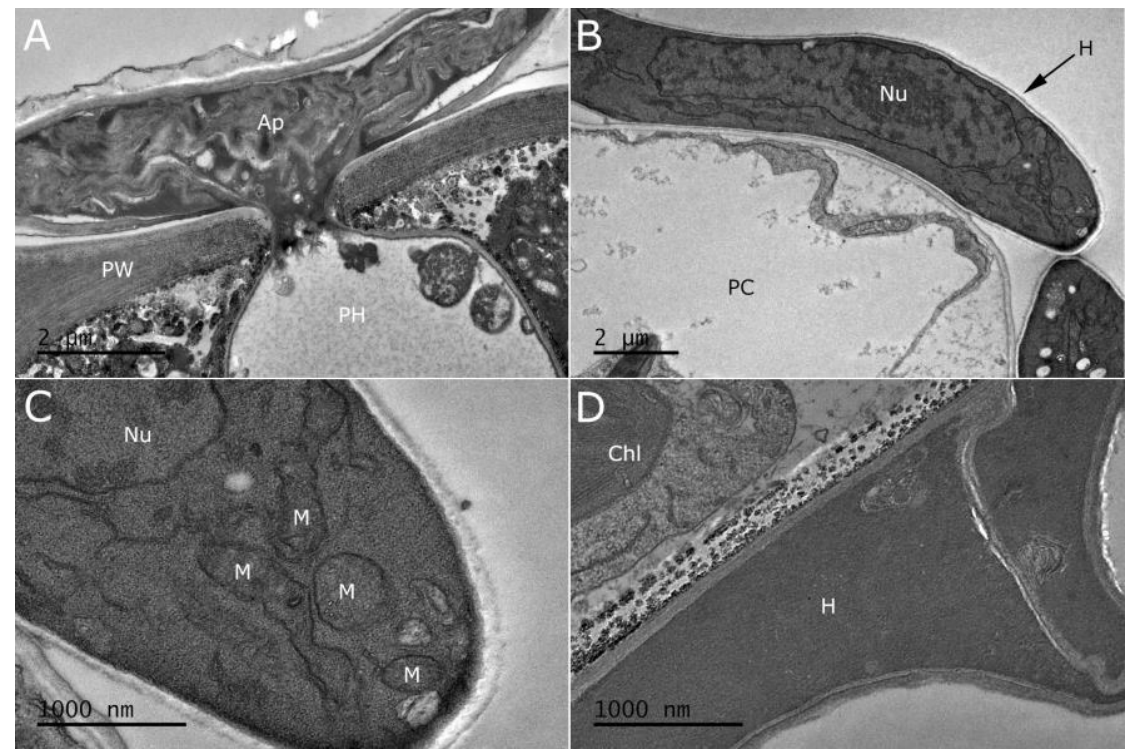

Figure 2. Transmission electron micrographs of resin-embedded sections of ASR-infected soybean leaves with and without fungicide treatment. A.) A single ASR appressorium in cross-section showing penetration of the plant cell wall (PW) and the formation of a primary infection hypha (PH). B.) A fungal hyphal cell $(\mathrm{H})$ directly adjacent to a plant cell $(\mathrm{PC})$, showing the ultrastructure of fungal cells in untreated leaves. C.) Higher magnification view of a fungal cell showing distinct organellar structures, including a nucleus $(\mathrm{Nu})$ and several mitochondria (M). D.) Hyphal cells inside a fungicide-treated soybean leaf, showing no evidence of organelles or recognizable cellular features. The ultrastructure of nearby chloroplasts $(\mathrm{Chl})$ in an adjacent plant cell appear healthy and unaffected. Ap = appressorium, $\mathrm{PW}=$ plant cell wall, $\mathrm{PH}=$ primary infection hypha, $\mathrm{PC}=$ plant cell, $\mathrm{H}=$ fungal hypha, $\mathrm{Nu}=$ nucleus, $\mathrm{M}=$ mitochondria, $\mathrm{Chl}=$ chloroplast.

\section{References}

[1] Goellner et al. 2020

[2] Miles et al. 2007 